\title{
Does unidirectional vesicular transport occur in retinal vessels?
}

\author{
T A GARDINER AND D B ARCHER
}

From the Department of Ophthalmology, The Queen's University, Belfast

SUMmaRY This paper challenges the hypothesis that the smooth $80 \mathrm{~nm}$ plasmalemmal caveolae found in abundance at the abluminal aspect of the endothelium in retinal blood vessels participate in a unidirectional vesicular transport mechanism. Evidence is presented which indicates that horseradish peroxidase, when introduced to the extracellular space of the retina via the vitreous body, may enter the intravascular compartment through junctional incompetence which occurs at or after enucleation of the eye. It is proposed that the plasmalemmal caveolae at the abluminal plasma membrane of endothelial cells in retinal blood vessels are static structures which facilitate the transport of small solutes and ions across the blood retinal barrier.

An unusual polarity has been described in the plasmalemmal caveolae in the vascular endothelial cells of the brain ${ }^{12}$ and retina. ${ }^{34}$ Caveolae are present in large numbers at the abluminal plasma membrane yet are relatively sparse at the luminal cell surface. The filling of such caveolae with intravitreal horseradish

Correspondence to T A Gardiner, MSc, Department of Ophthalmology, The Queen's University of Belfast, Eye and Ear Clinic, Royal Victoria Hospital, Belfast BT12 6BA, Northern Ireland. peroxidase (HRP) together with a simultaneous occurrence of the tracer in the lumina of the same vessels led the authors of a recent study ${ }^{4}$ to conclude that 'unidirectional vesicular transport' occurs in retinal blood vessels, that is, from the extracellular space to the intravascular compartment. Nevertheless, 'in transit' vesicles were not demonstrated, and no corresponding complement of plasmalemmal caveolae were identified at the luminal plasma membrane.

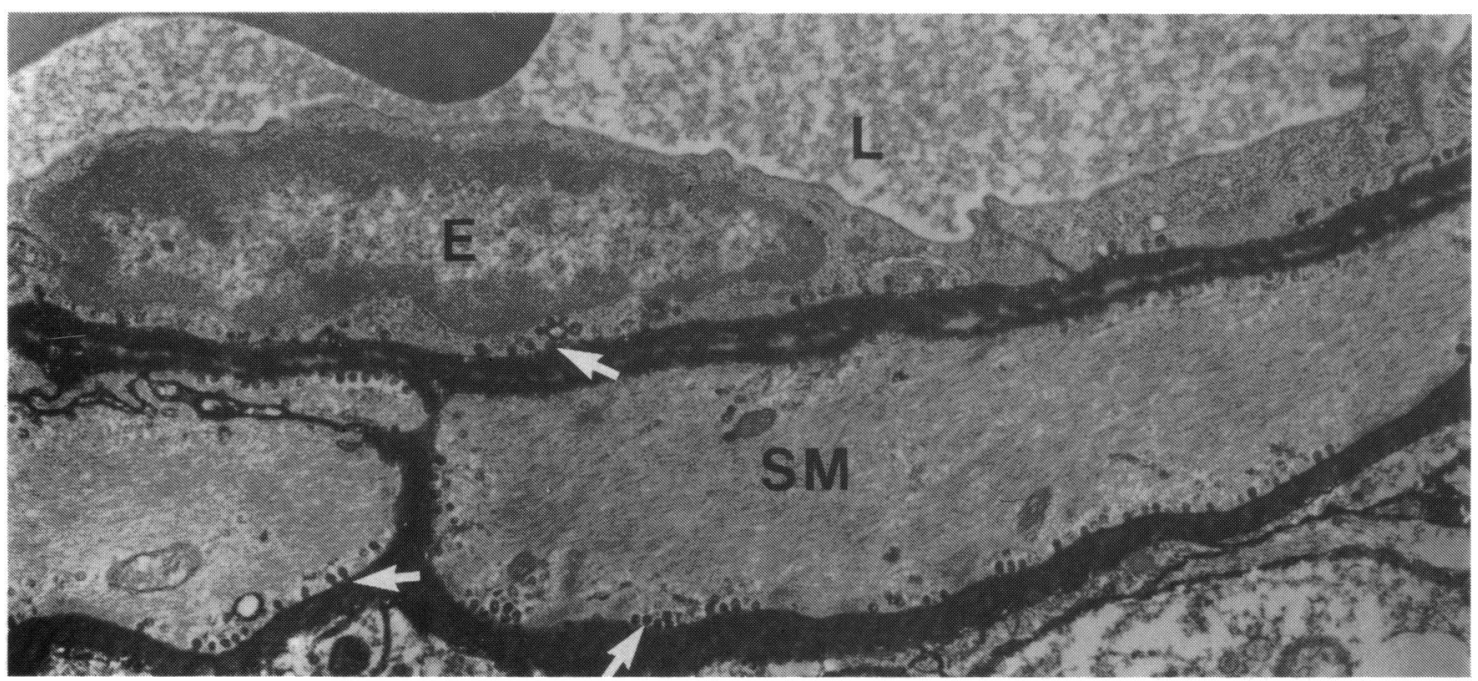

Fig. 1 Retinal artery (rat): intravitreal horseradish peroxidase (immersion fixation). Peroxidase reaction product fills the basement membranes of a retinal artery. The sarcolemmal caveolae of the smooth muscle cells (SM) and the abluminal plasmalemmal caveolae of the endothelium (E) are outlined by the tracer (arrows). Lumen (L). ( $\times 12000)$. 
Westergaard and Brightman' suggested that the abluminal plasmalemmal caveolae of cerebral vessels were static structures. This view was confirmed by Broadwell et al., ${ }^{2}$ who found that the abluminal caveolae of endothelial cells in the cerebral vessels of the mouse did not transport intraventricular horseradish peroxidase even after 24 hours' exposure to the tracer. Accordingly, this study was undertaken to elucidate the function of the plasmalemmal caveolae at the abluminal surface of the retinal vascular endothelium.

\section{Materials and methods}

Three male Wistar albino rats were anaesthetised by intraperitoneal injection of sodium pentobarbital. Horseradish peroxidase (Sigma type VI) dissolved in Hams F10 tissue culture medium (10 mg HRP/ml) was injected into the vitreous body of both eyes in each animal. $50 \mu \mathrm{l}$ of peroxidase solution was injected into the vitreous body via the peripheral retina. After $30 \mathrm{~min}$ the eyes were enucleated and fixed by immersion in $2.5 \%$ gluteraldehyde in $0.1 \mathrm{M}$ cacodylate buffer. Areas of retina remote from the injection site were selected for horseradish peroxidase demonstration and processed as previously described. ${ }^{5}$

\section{Results}

Thirty minutes after intravitreal injection of horseradish peroxidase its reaction product was found throughout the entire extracellular space of the retina. The tracer had penetrated the basement membranes of both large and small blood vessels (Figs. 1 and 2) and filled the abluminal plasmalemmal caveolae of the endothelial cells. The caveolae usually occurred as single units, although composite structures were often seen (Fig. 3). Labelled vesicular images were seen adjacent to the abluminal plasma membranes of the endothelial cells, but no 'in transit' vesicles were found in either the central or apical cytoplasm (Fig. 3). At the time of examination (30 min postintravitreal injection of HRP) the multivesicular bodies of the endothelial cells showed no evidence of peroxidase incorporation (Fig. 3). The sarcolemmal caveolae of vascular smooth muscle cells (Figs. 1 and 3 ) filled uniformly with peroxidase, though there was no evidence of tracer internalisation. A large proportion of the vessels showed HRP reaction product within the fixed plasma in the vessel lumen.

The intercellular clefts bounded by the tight junctions of the endothelial cells were usually free of peroxidase (Fig. 3). However, it was possible to find vessels where the tight junctions had been breached and where peroxidase was present throughout the intercellular clefts (Fig. 4) and in the vessel lumen (Figs. 2, 4, and 5). 'Tight' and 'leaky' junctions could be found in the same vascular profile (Fig. 6). However, the observed sites of junctional incompetence were discrete, and even in these regions the adjacent plasma membranes showed no gross separation (Fig. 4).

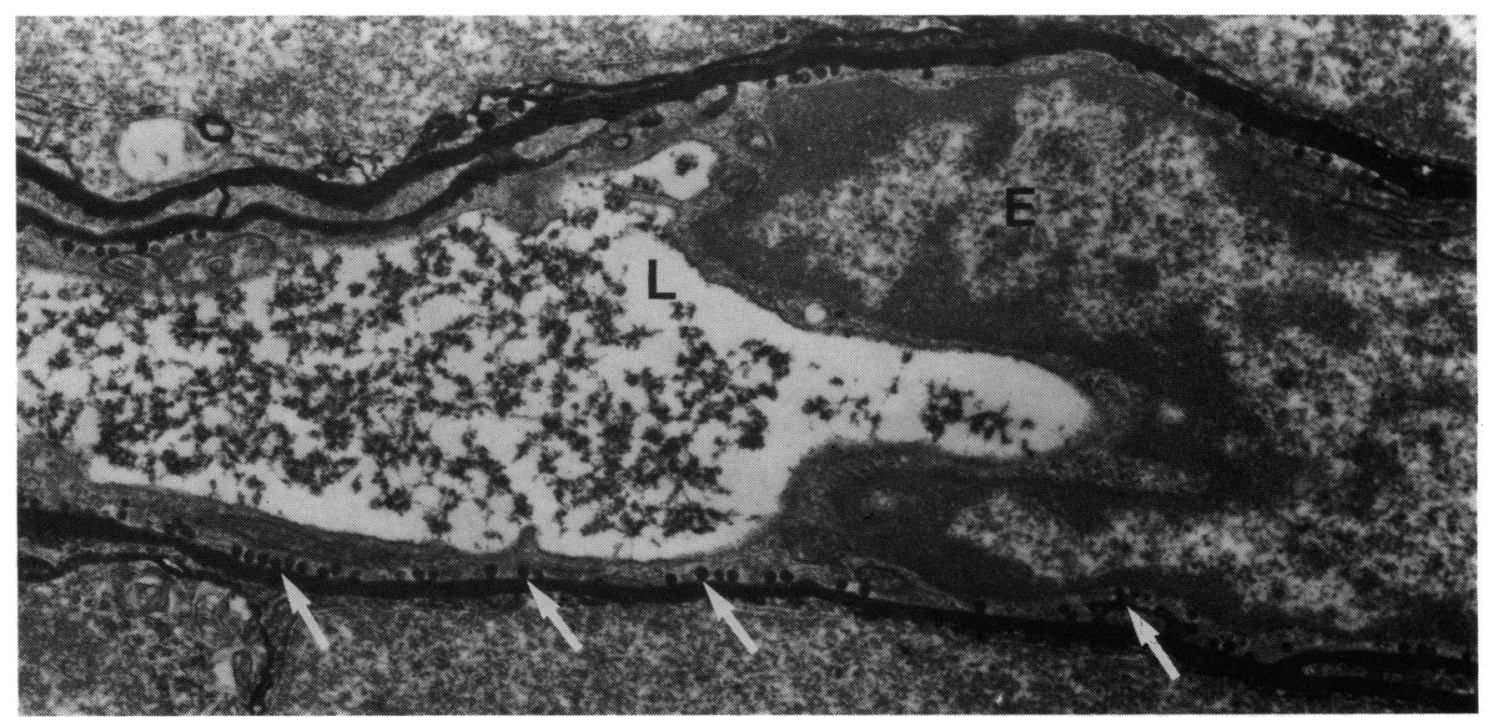

Fig. 2 Retinal capillary (rat): intravitreal horseradish peroxidase. Peroxidase reaction product fills the vascular basement membranes and outlines numerous abluminal plasmalemmal caveolae (white arrows) in the endothelium (E). The plasma in the vessel lumen is also stained by peroxidase reaction product. Lumen $(\mathrm{L}) .(\times 15000)$. 


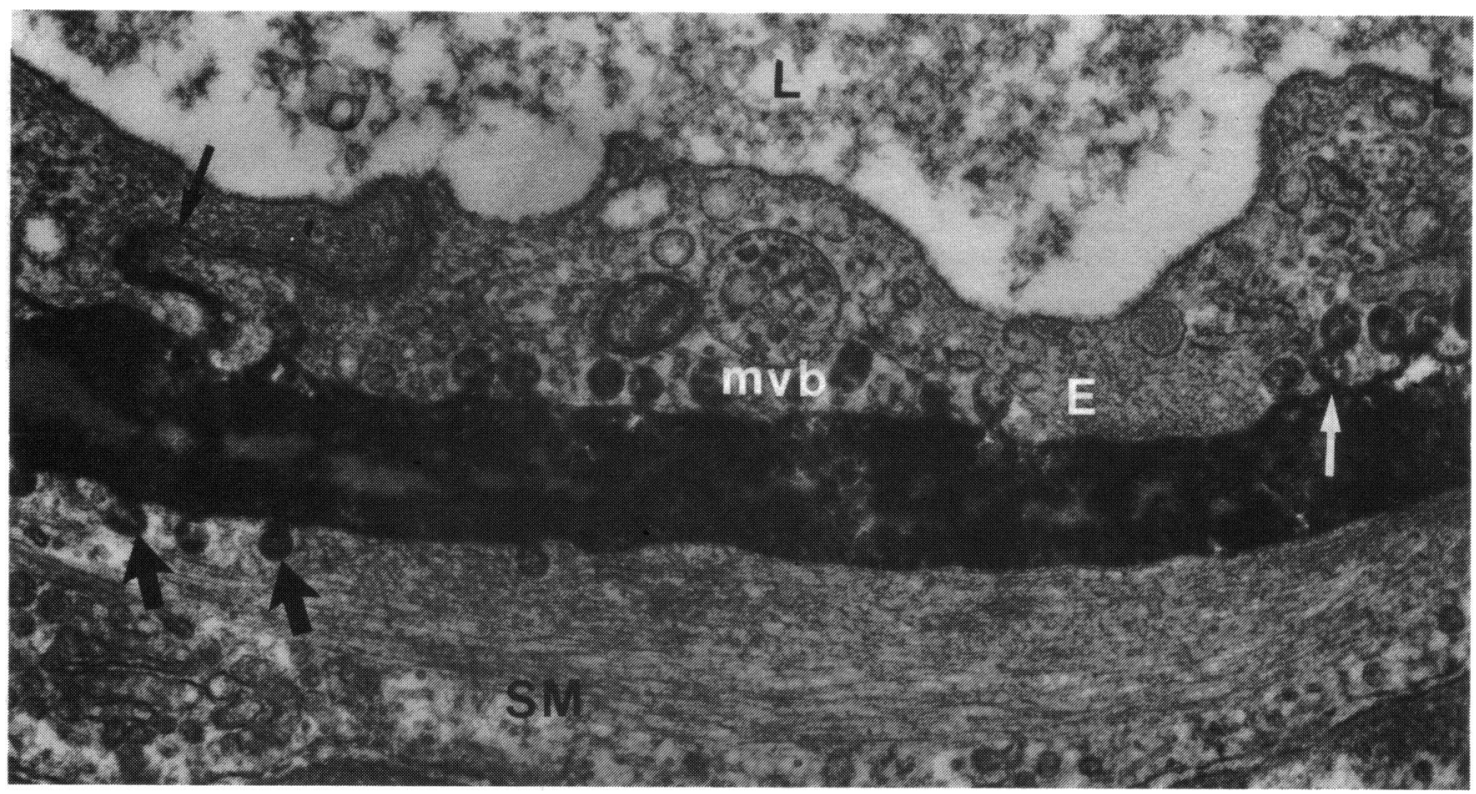

Fig. 3 Retinal artery (rat): intravitreal horseradish peroxidase, immersion fixation. Bilobular vesicular structures associated with the abluminal plasma membrane of endothelial cell (E) are filled by peroxidase reaction product (white arrow), but a nearby multivesicular body ( $\mathrm{mvb}$ ) shows no evidence of tracer ingestion. Tracer does not penetrate beyond the first tight zone of the junctional complex (thin black arrow). The sarcolemmal caveolae (thick black arrows) of a smooth muscle cell (SM) are stained with peroxidase reaction product. Lumen $(\mathrm{L}) .(\times 45500)$.

\section{Discussion}

The preponderance of abluminal over luminal plasmalemmal caveolae in the endothelia of cerebral vessels was first recorded by Westergaard and Brightman,' and a similar situation has recently been described in retinal vessels. ${ }^{34}$ To test the possibility that such caveolae play a role in macromolecular transport from the extracellular space of the retina to the intravascular compartment they were presented with a traceable protein (horseradish peroxidase) via the vitreous body. Peyman et al. ${ }^{6}$ noted that intravitreal horseradish peroxidase fully penetrated the rabbit retina within 15 minutes. Accordingly we considered that 30 minutes was an adequate time for intravitreal horseradish peroxidase to penetrate to the blood vessels of the thinner rat retina and permit possible endocytosis at the abluminal surface of the endothelium. In none of the retinal vessels examined was there evidence of vesicular transport or internali-

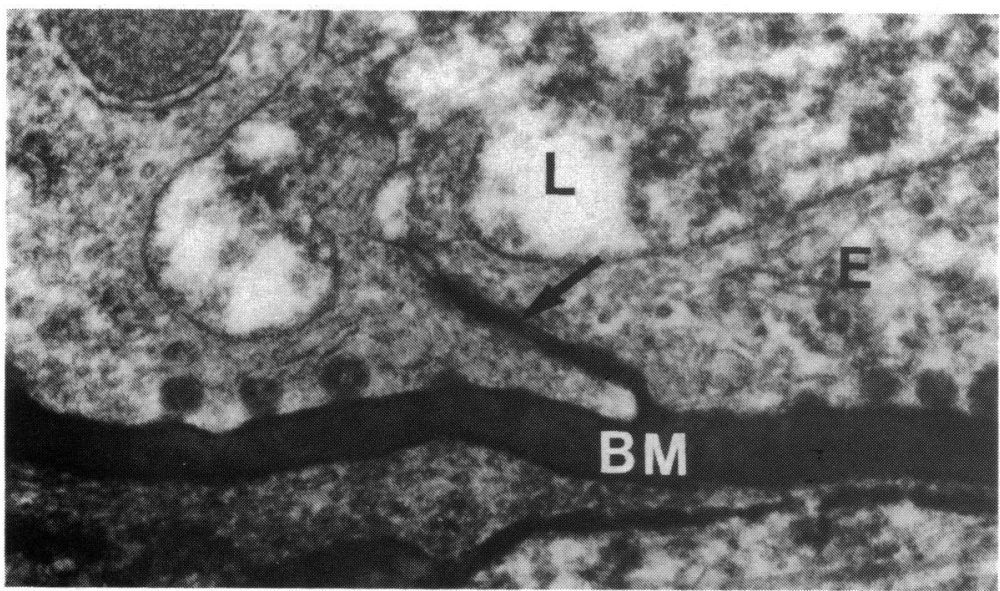

Fig. 4 Retinal capillary (rat): intravitreal horseradish peroxidase. Peroxidase has passed through the cell junctions in this vessel (arrow) and is present in the lumen $(\mathrm{L})$. Endothelial cell (E). Basement membrane $(\mathrm{BM}) .(\times 50000)$. 


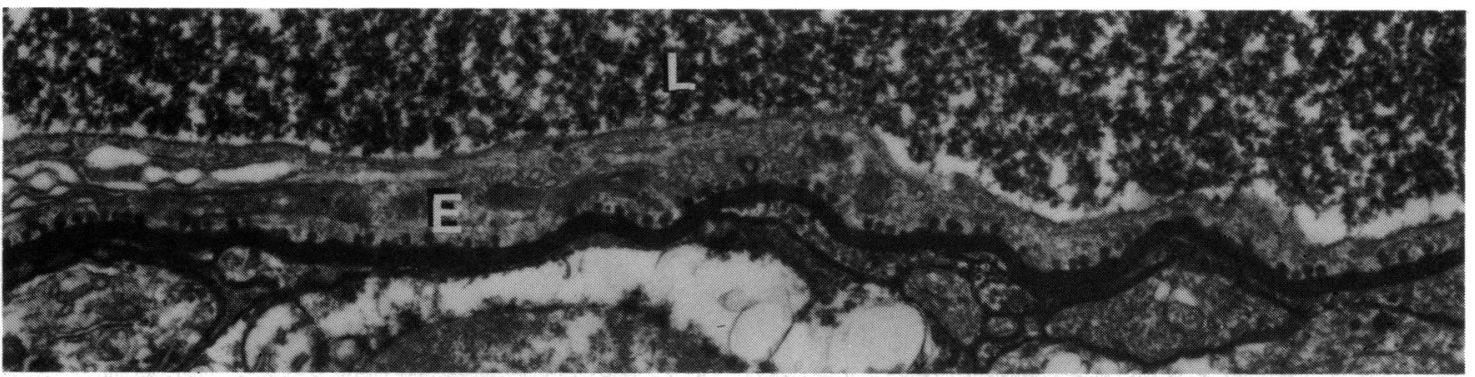

Fig. 5 Retinal capillary (rat): intravitreal horseradish peroxidase. Peroxidase reaction product stains the basement membrane and abluminal plasmalemmal caveolae of the endothelium $(\mathrm{E})$. Tracer is present in the lumen $(\mathrm{L}) .(\times 15000)$.

sation of peroxidase by the abluminal plasma membranes of the endothelial cells. If transcellular vesicular transport had been in progress at the time of fixation, it is almost certain that a few labelled vesicles would have been seen either 'in transit' or at the luminal plasma membrane. These results support the suggestion of Westergaard and Brightman, ${ }^{1}$ who considered that the abluminal plasmalemmal caveolae of cerebral vessels were static structures involved more with ion fluxes than macromolecular transport.

Ultrathin serial sectioning of frog mesenteric capillaries $^{7}$ and rat heart capillaries ${ }^{8}$ has revealed that the plasmalemmal caveolae of vascular endothelial cells often form racemose invaginations deep into the endothelial cell cytoplasm. Conventional thin sections give the false impression that such structures are free in the cytoplasm while in reality they are still connected to the cell surface. In the present study the abluminal caveolae did not appear to form particularly deep invaginations, as 'vesicle' images were never encountered at a depth at which direct communication with the cell surface was not a likely possibility.

The presence of numerous abluminal caveolae in the endothelium of cerebral vessels and the danger of misrepresenting them as transport vesicles was highlighted by Broadwell et al. ${ }^{2}$ These workers found no evidence of vesicular transport of intraventricular horseradish peroxidase even after 24 hours' exposure to the tracer.

The results of the present study are contrary to those of a recent report by Raviola and Butler, ${ }^{4}$ who described a 'unidirectional vesicular transport mechanism in retinal vessels' based on observations of intravitreally administered horseradish peroxidase within the retina. The authors observed HRP reaction product staining the fixed plasma within the lumina of the retinal vessels and attributed it to the activity of a vesicular transport mechanism arising from the abluminal plasmalemmal caveolae. The authors contended that the absence of a correspond-
Fig. 6 Retinal arteriole (rat): intravitreal horseradish peroxidase. Two junctional complexes $(\mathrm{J})$ are present at the lateral aspects of a single endothelial cell $(\mathrm{E})$. The tight junction at one end of this cell has leaked at a point outside the plane of section and admitted peroxidase to the intercellular cleft (arrow). In regions where the abluminal plasmalemma is obliquely sectioned the associated caveolae appear as 'vesicles' (white arrows). Lumen (L), Smooth muscle cell (SM). $(\times 40000)$.

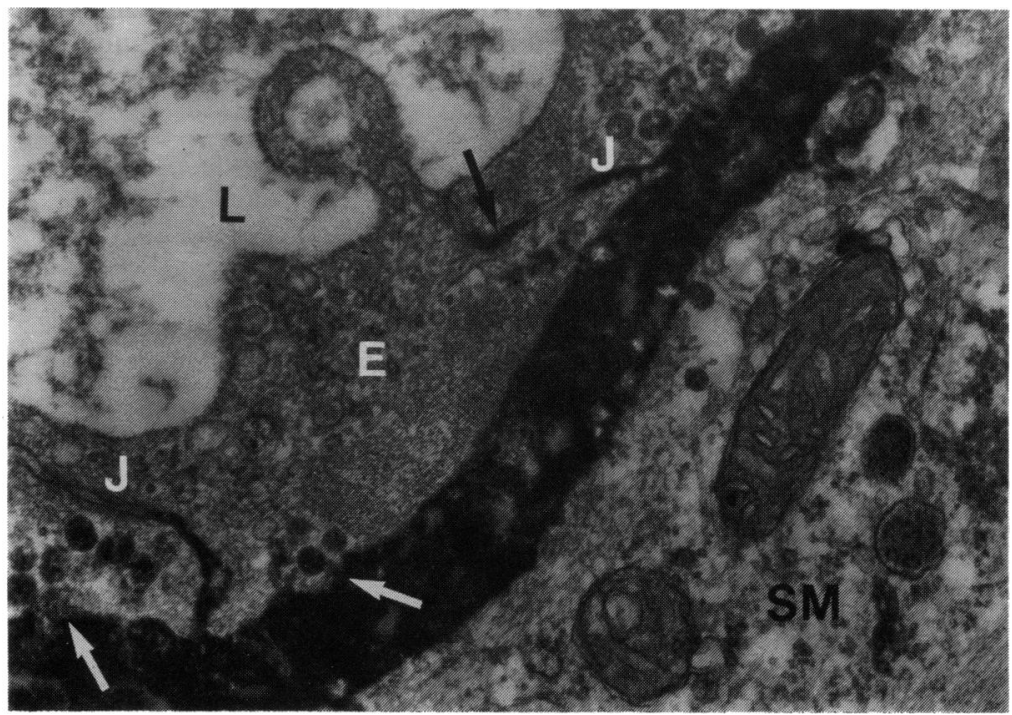


ing number of plasmalemmal vesicles at or even approaching the luminal surface of the endothelium did not 'seem to represent a cogent objection' to the notion that the abluminal plasmalemmal caveolae give rise to transport vesicles. We suggest that the failure to demonstrate a progression of labelled vesicles or vesicle derived structures across the endothelium presents a major difficulty to the hypothesis that vesicular transport occurs in these vessels. Raviola and Butler did publish an electron micrograph of two peroxidase labelled caveolae which were thought to be discharging their contents at the luminal surface of the endothelium. However, they did not mention that peroxidase arriving in the vessel lumen by whatever route is likely to stain the luminal glycocalyx of the endothelium, including that on the interior of the plasmalemmal caveolae. ${ }^{9}$ The glycocalyx or anionic surface coat is present around most cells, ${ }^{10}$ and its presence is well recognised in vascular endothelia." Sigma type II HRP as used by Raviola and Butler is known to be a mixture of several different isoenzymes, some of which are cationic and as such have an affinity for the polyanions of the glycocalyx. Their published micrograph clearly shows positive staining by adsorbed peroxidase within the glycocalyx of the luminal plasma membrane which is continuous with the caveolae said to be 'discharging'. Furthermore, the peroxidase stained layer within the caveolae is clearly identical to that on the plasmalemma proper. The tracer which was present in the vessel lumen in the study by Raviola and Butler and in the present investigation was obviously the result of a post-mortem phenomenon, as immersion fixation was used in both cases. Accordingly the 'unidirectional vesicular transport' hypothesis presented by Raviola and Butler as a physiological mechanism seems unlikely.

The present study has shown that the 'tightness' of the endothelial cell junctions in the retinal vessels may be functional, that is, be dependent on a normal relationship between intravascular and tissue pressure. The fall in intravascular pressure at enucleation (when the central retinal artery and vein were severed) may have allowed the relatively high tissue pressure to force peroxidase laden fluid from the extracellular space through the cell junctions and into the vessel lumen. As soon as the central retinal artery and vein are severed, any pressure applied to the globe in subsequent handling will tend to transfer fluid from the extracellular space of the retina to the intravascular compartment. It is therefore suggested that in experiments of this type the intraocular pressure should be reduced to zero before interrupting the retinal blood supply.

Preliminary experiments on monkeys in this laboratory suggest that intravitreal peroxidase is not found in the intravascular compartment if the intraocular pressure is reduced before cutting the optic nerve. Although Raviola and Butler illustrated an intact cell junction in a vessel showing peroxidase in the lumen, the present study has shown that competent and incompetent junctions may be present in the same vessel. Therefore it does not appear to be necessary for all or even a large proportion of the cell junctions within the retinal vessels to leak in order to fill considerable segments of the vascular bed with peroxidase laden fluid. Furthermore, as the transjunctional 'leaks' observed in the present study were subtle and not noticed at all by Raviola and Butler in their study, it seems that a relatively small number of incompetent vessels may have been responsible for the presence of intravascular peroxidase. It is therefore not surprising that attempts to arrest the transport of HRP from the extracellular space to the vessel lumen by cooling the eye and using metabolic poisons should have failed in the study by Raviola and Butler.

If the abluminal plasmalemmal caveolae are not concerned with the transport of macromolecules, then it remains to be determined just what their precise functions are. In a general sense it seems likely that their role may be analagous to those of the sarcolemmal caveolae of smooth muscle cells, ${ }^{12-14}$ which greatly increase the surface area of the plasma membrane for more subtle membrane functions-for example, simple or facilitated diffusion or active transport. Caveolae would provide a stable microenvironment conducive to the operation of specific transport systems within the turbulent environment of the vascular wall. It is relevant that enzyme systems associated with active transport have been demonstrated within endothelial plasmalemmal caveolae. ${ }^{15}{ }^{16}$ Furthermore the membrane associated ATPase activity of retinal ${ }^{17}$ and cerebral ${ }^{18}$ vascular endothelia shows a distinct polarity, being located at the abluminal surface. Cunha-Vaz and Maurice ${ }^{19}$ have demonstrated the ability of the retinal blood vessels to pump organic anions out of the retina against a concentration gradient. We suggest that this 'pump' and perhaps other specific extrusion systems for metabolic wastes are likely to be located at the abluminal plasma membrane of the retinal endothelial cells and that the abluminal plasmalemmal caveolae represent the structural facility required to accommodate such systems.

\section{References}

1 Westergaard E, Brightman M. Transport of proteins across normal cerebral arterioles. J Comp Neurol 1973; 152: 17-44.

2 Broadwell RD, Balin BJ, Salaman M, Kaplan RS. Brain blood barrier? Yes and no. Proc Natl Acad Sci USA 1983; 80: 7352-6. 
3 Archer DB. Retinal neovascularization. Trans Ophthalmol Soc UK 1983; 103: 2-27.

4 Raviola G, Butler JM. Unidirectional vesicular transport mechanism in retinal vessels. Invest Ophthalmol Vis Sci 1983; 24: 1465-74.

5 Gardiner TA, Archer DB. Endocytosis in the retinal and choroidal microcirculation. Br J Ophthalmol in press.

6 Peyman GA, Spitznas M, Straatsma BR. Peroxidase diffusion in the normal and photocoagulated retina. Invest Ophthalmol Vis Sci 1971; 10: 181-9.

7 Frøkjaer-Jensen J. Three-dimensional organization of plasmalemmal vesicles in endothelial cells. An analysis by serial sectioning of frog mesenteric capillaries. J Ultrastruct Res 1980 ; 73: 9-20.

8 Bundgaard $\mathrm{M}$, Hagman $\mathrm{P}$, Crone $\mathrm{C}$. The three-dimensional organization of plasmalemmal vesicular profiles in the endothelium of rat heart capillaries. Microvasc Res 1983; 25: 358-68.

9 Luft JH. Fine structure of the capillary and endocapillary layer as revealed by ruthenium red. Fed Proc 1966; 25: 1773-83.

10 Rambourg A, Leblond CP. Electron microscopic observations on the carbohydrate-rich cell coat present at the surface of cells in the rat. J Cell Biol 1967; 32: 27-53.

11 Luft JH. The structure and properties of the cell surface coat. Int Rev Cytol 1976; 45: 291-382.

12 Gabella GI. Structure of smooth muscles. In: Bülbring E,
Brading SF, Jones AW, Tomita T, eds. Smooth muscle. London: Arnold, 1981: 3-5.

13 Popescu LM, Diculescu I, Zelek U, Ionescu N. Ultrastructural distribution of calcium in smooth muscle cells of guinea-pig Taenia coli. Cell Tissue Res 1974; 154: 357-78.

14 Popescu LM. Conceptual model of the excitation-contraction coupling in smooth muscle: the possible role of surface microvesicles. Studia Biophysica (Berlin) 1974; 44: 141-53.

15 Marchesi VT, Barrnett RJ. The demonstration of enzymatic activity in pinocytotic vesicles of blood capillaries with the electron microscope. J Cell Biol 1963; 17: 547-56.

16 Ryan US. Structural bases for metabolic activity. Ann Rev Physiol 1982; 44: 223-39.

17 Marchesi VT, Barrnett RJ. The localization of nucleotide phosphatase activity in different types of small blood vessels. $J$ Ultrastruct Res 1964; 10: 103-15.

18 Betz AL, Firth AJ, Goldstein GW. Polarity of the blood-brain barrier: distribution of enzymes between the luminal and antiluminal membranes of brain capillary endothelial cells. Brain Res 1980; 192: 17-28

19 Cunha-Vaz JG, Maurice DM. The active transport of fluorescein by the retinal vessels and the retina. J Physiol (Lond) 1967; 191: $467-86$.

Accepted for publication 15 August 1985. 